\title{
Phosphoribosyl pyrophosphate synthetases 2 knockdown inhibits prostate cancer progression by suppressing cell cycle and inducing cell apoptosis
}

\author{
Hui Qiao ${ }^{3^{*}}$, Xiao Tan ${ }^{*}$, Dao-jun Lv ${ }^{1,2^{*}}$, Rong-wei Xing5, Fang-peng Shu², Chuan-fan Zhong2, Chun Li ${ }^{3}$, \\ Ya-guang Zou ${ }^{\bowtie}$, Xiang-ming $\mathrm{Mao}^{1,2}$ \\ 1. Department of Urology, Nanfang Hospital, Southern Medical University, 510515, Guangzhou, Guangdong Province, China. \\ 2. Department of Urology, Zhujiang Hospital, Southern Medical University, 510282, Guangzhou, Guangdong Province China. \\ 3. Nursing Department, Nanfang Hospital, Southern Medical University, 510515, Guangzhou, Guangdong Province, China. \\ 4. Department of Stomatology, Nanfang Hospital, Southern Medical University, 510515, Guangzhou, Guangdong Province, China. \\ 5. Department of Urology, the Affiliated Weihai Second Municipal Hospital of Qingdao University, 264200, Weihai, Shandong Province, China. \\ "These authors contributed equally to this work.
}

$\triangle$ Corresponding author: Xiang-ming Mao, Department of Urology, Nanfang Hospital, Southern Medical University. 1838 Guangzhou Avenue North, Tonghe, Baiyun District, Guangzhou, Guangdong Province, China; Phone: +86 13802503635; Fax: +86-20-61641047; Email: mxm631221@126.com. Or, Ya-guang Zou, Department of Stomatology, Nanfang Hospital, Southern Medical University. 1838 Guangzhou Avenue North, Tonghe, Baiyun District, Guangzhou, Guangdong Province, China; Phone: +86-18620050609; Fax: +86-20-61641047; Email: 18620050609@163.com.

(c) The author(s). This is an open access article distributed under the terms of the Creative Commons Attribution License (https://creativecommons.org/licenses/by/4.0/). See http://ivyspring.com/terms for full terms and conditions.

Received: 2019.06.07; Accepted: 2019.10.11; Published: 2020.01.01

\begin{abstract}
Phosphoribosyl pyrophosphate synthetases 2 (PRPS2) protein function as nucleotide synthesis enzyme that plays vital roles in cancer biology. However, the expression profile and function of PRPS2 in prostate cancer (PCa) remain to be identified. Here we investigated the expression of PRPS2 protein in human PCa and paired normal tissues by immunohistochemistry, meanwhile the regulatory effects on cell proliferation, apoptosis and growth of xenograft tumors in nude mice were evaluated in PCa cells with PRPS2 depletion. Moreover, the signaling pathways were also explored by western blot analysis and quantitative polymerase chain reaction assays. We found that PRPS2 was dramatically upregulated in prostate adenocarcinoma tissues in comparison with normal tissues, and that increased PRPS2 was linked intimately to advanced clinical stage and PT status. Functional experiments showed that knockdown of PRPS2 significantly suppressed cell growth both in vitro and in vivo. In addition, depletion of PRPS2 induced $\mathrm{G}_{1}$ phase cell cycle arrest and elevated cell apoptosis. Silencing of PRPS2 resulted in the decreased expression of $\mathrm{Bcl}-2$ and cyclinDl and increased levels of Bax, cleavage of caspases-3, caspases-9 and PARP. Furthermore, we also detected PRPS2 expression was significantly induced after DHT treatment, which implied the important role of PRPS2 in oncogenesis of PCa. Taken together, our findings elucidated that PRPS2 may be a potential novel candidate for PCa therapy.
\end{abstract}

Key words: apoptosis, Phosphoribosyl pyrophosphate synthetase 2, proliferation, prostate cancer.

\section{Introduction}

Prostate cancer (PCa) is the fifth leading causes of cancer-related death and the most common human carcinoma among men worldwide [1]. Androgen deprived therapy (ADT) or platinum-based chemotherapy or other systemic treatment has been proved to partial effectively to alleviate symptoms for advanced prostate adenocarcinoma patients; however, the overall survival benefit is still limited
[2]. A growing number of evidence highlights that exceptional overexpression of apoptosis resistant proteins, and dysregulation of proliferation-related genes is obsessed in the advance of PCa [3, 4]. Whereas, most of the specific elements in the tumorigenesis of PCa remain poorly understood. Hence, it is imperative to find biomarkers that might improve the therapeutic capacity of PCa. 
Human phosphoribosyl pyrophosphate synthases isoform 2 (PRPS2) is a key rate-limiting enzyme in the purine biosynthesis pathway, which catalyzes the irreversible conversion of ribose 5-phosphate to phosphoribosyl pyrophosphate (PRPP) with the help of $\mathrm{Mg}^{2+}$-ATP $[5,6]$. Multiple researches declared that PRPS isoforms played quiet different roles between physiology and pathological state, but the mechanisms of functional difference between human PRPS2 and PRPS1 are still uncovered. A recent study showed a special role of PRPS2 in Myc-driven tumorigenesis and identified PRPS2 as a target protein that can manipulate cancer cells both in nucleotide biosynthesis and metabolic rate aspects [7]. Interestingly, PRPS2 was reported to contain a consensus pyrimidine-rich translational element (PRTE) motif within its 5' UTR that enables Myc directly increase nucleotide biosynthesis via translational regulation mechanisms to the enhanced protein synthesis of cancer cells $[7,8]$. Notably, our previous study revealed that the expression of PRPS2 correlated with sertoli-cell only syndrome via inhibits cell apoptosis [9]. All of these indicated the important role of PRPS2 in the onset and progression of multiple human diseases. However, the detailed function and mechanism of PRPS2 in PCa are still uncertain.

Here, our research was aimed to investigate the precise biological characters of PRPS2 in the development and progression of PCa. Our findings suggested that PRPS2 was frequently upregulated in PCa tissues compared with that in adjacent normal tissues. Additionally, the upregulation of PRPS2 was related to PCa malignant progression. Furthermore, silencing of PRPS2 triggered cell cycle arrest and caspase3-dependent apoptosis. Therefore, PRPS2 may serves as a tumor promoter and a potential new therapeutic target for PCa.

\section{Materials \& Methods}

\section{Clinical specimens}

The tissue microarray (TMA) was constructed as described previously [10], using archived pathological formalin-fixed, paraffin-embedded specimens collected from localized PCa patients who underwent radical prostatectomy (RP) or biopsy and confirmed by pathology at the Nanfang Hospital between January 2013 and June 2018. The detailed criteria for the inclusion and exclusion of patients were as follow: Inclusion criteria, 1. Patients diagnosed with PCa before surgery in accordance with biopsy pathological diagnosis; 2. Patients have written the informed consent to collect the specimens. Exclusion criteria, 1. These patients with other malignant disease or a second primary tumor; 2 . These PCa patients who received preoperative chemotherapy or radiotherapy before surgery; 3 . HIV or syphilis positive patients. All our experimental protocols were authorized by the Ethics Committee of the Southern Medical University Nanfang Hospital. The detailed clinic parameters of enrolled patients, including the age, gender, tumor location, tumor differentiation and tumor node metastasis (TNM) classification, were archived and exhibited in Table S1. The pathological diagnoses of enrolled patients were verified by two different pathologists based on the WHO grading system.

\section{Immunohistochemistry (IHC) analyses}

IHC assay was carried on paraffin-embedded tissue sections to determine PRPS2 (1:50, \#NBP1-31435, Novus) and Ki-67 (1:200, \#ab16667, Abcam) protein expression. The avidin-biotinperoxidase method was adopted to confirm the location and the relative expression level of the PRPS2. Briefly, tissue slides were deparaffinized with xylene and rehydrated with ethanol, and subjected to boiled retrieval in a sodium citrate buffer for $10 \mathrm{~min}$. Sections were subsequently immersed in $3 \% \mathrm{H}_{2} \mathrm{O}_{2}$ for $30 \mathrm{~min}$ to quench endogenous tissue peroxidase activity. Subsequently, slides were incubated with primary antibodies overnight at $4{ }^{\circ} \mathrm{C}$, followed by incubation with the biotin-linked anti-Rabbit secondary antibody (Zhongshan Biotech, China) in combination with the diaminobenzidine (DAB) complex. Slides were counterstained with hematoxylin, then dehydrated and mounted with glass coverslips according to standard protocol. The positive staining intensity of PRPS2 was scored into four categories: 0 , negative; 1 , weakly positive; 2 , intermediately positive; and 3 , strongly positive. The percentage of PRPS2 positive cells was scored as four categories: 0 , no staining; 1 : $<25 \%$ cells; 2 : $25 \%-75 \%$ cells; and 3: $>75 \%$ cells. Total protein expression score (ranging from 0 to 9) of a sample was obtained by the multiplication of the intensity and percentage scores, as previously described [11]. The staining pattern of TMA was scored based on the total protein expression scores as follows: total protein expression score: $0,-$; $1-3,+; 4-6,++$; and 6-9, +++. We subsequently divided our cases into two groups using total protein expression scores: cases with total expression score 0 $3(-/+)$ was regarded as the low expression group, and cases with total expression score $4-9(++/+++)$ was regarded as the high expression group. Sections were visualized under a microscope (Olympus, Japan).

\section{Cell cultures}

Human PCa cell lines PC3, DU145, C4-2, LNCaP 
and the non-malignant immortalized human prostate epithelial cell line RWPE-1 were obtained from Chinese Academy of Sciences cell bank (Shanghai, China). All PCa cell lines were routinely cultured in RPMI-1640 (Gibco, Grand Island, NY, USA) containing 10\% fetal bovine serum (Life Technologies, Monza, Italy) and 1\% penicillin/streptomycin (Life Technologies). RWPE-1 cells were grown in Keratinocyte Serum Free Medium (KSFM) (Gibco, No. 10724-011) supplemented with $50 \mathrm{mg} / \mathrm{mL}$ bovine pituitary extract (Gibco, No. 13028-014), $5 \mathrm{ng} / \mathrm{mL}$ epidermal growth factor (EGF) (Gibco, No. 10450-013), and 1\% antibiotic-antimycotic solution (Gibco, No. 15240062). Cells were incubated at $37^{\circ} \mathrm{C}$ in a humidified atmosphere of $5 \% \mathrm{CO}_{2}$. $\mathrm{LNCaP}$ cells were maintained in Phenol Red-free RPMI 1640 (GIBCO, No. 11835-030) contained with 10\% charcoal-dextran-stripped FBS for $72 \mathrm{~h}$ before androgen stimulation, then double hydrogen testosterone (DHT) was added to the culture medium.

\section{RNA extraction and real-time qRT-PCR}

Total RNA was isolated with TRIzol reagent (TaKaRa) according to the manufacturer's instructions. Complementary DNA was reverse-transcribed using Prime Script RT reagent Kit (TaKaRa Biomedical Technology, Dalian, China). Quantitative Real-time PCR (qRT-PCR) analysis was conducted using the SYBR ${ }^{\circledR}$ Green PCR Master Mix (Toyobo, Osaka, Japan). The specific primers set for human PRPS2 were: 5'-AGCTCGCATCAGGAC CTGT-3' (forward) and 5'- ACGCTTTCACCAAT CTCCACG-3' (reverse), and GAPDH primers were 5'-CCAGGTGGTCTCCTCTGACTTC-3' (forward) and 5'-GTGGTCGTTGAGGG CAATG -3' (reverse). All data analyses were managed using the ABI 7500 Fast Analyzer (Life Technologies Corp.). Data were gathered from three biological and technical replicates then normalized to GAPDH expression levels by using the $2^{-\Delta \Delta \mathrm{Ct}}$ method.

\section{Western blotting analysis}

Cells were collected in PBS and lysed in RIPA lysis buffer containing proteinase inhibitors (\#KGP250, KeyGEN BioTECH, Nanjing, China) according to the manufacturer's protocols. Lysates were vortexed, prepared on ice for $30 \mathrm{~min}$, and centrifuged at $10,000 \times \mathrm{g}$ for $30 \mathrm{~min}$ at $4{ }^{\circ} \mathrm{C}$. The supernatants were transferred into new tubes, and protein concentrations were measured by BCA assay (\#KGP906, KeyGEN BioTECH). Equal total of $30 \mu \mathrm{g}$ cell protein was loaded onto sodium dodecyl sulphate-polyacrylamide electrophoresis gels and electric-transferred onto PVDF membranes (Millipore, Billerica, MA, USA). All membranes were blocked with a Tris/saline solution containing $5 \%$ non-fat milk and $0.1 \%$ Tween-20 for $1 \mathrm{~h}$ and probed with specific primary antibody: PRPS2 (\#NBP1-31435, rabbit, Novus), Caspase-3 (\#9665, rabbit, Cell Signaling Technology), Cleaved Caspase-3 (\#9664, Cell Signaling Technology), PARP (\#9542, Cell Signaling Technology), Cleaved PARP (\#5625, Cell Signaling Technology), Cleaved Caspase-9 (\#52873, Cell Signaling Technology), Caspase-9 Mouse mAb (\#9508, Cell Signaling Technology), cyclin D1(\#2978, Cell Signaling Technology), p27 Kip1(\#3686, Cell Signaling Technology), CDK4 (\#12790, Cell Signaling Technology), anti-p53 (ab131442, Abcam), $\beta$-actin Mouse mAb (\#60008-1-Ig, Proteintech) and a-Tubulin rabbit mAb (\#11224-1-AP, Proteintech) antibodies. After extensive washing, membranes were incubated with secondary conjugated to horseradish peroxidase (1:10,000; Cell Signaling) for $1 \mathrm{~h}$ at room temperature. Proteins were visualized and detected using ECL kit (Thermo; Rockford, IL).

\section{siRNA transfection and establishment of stably transfected cell line}

Three siRNAs targeting PRPS2 (si-PRPS2-1, 2, 3) and negative control siRNA (NC) with no definite target were purchased from by RiboBio Company (Guangzhou, China). The sequences of siRNA oligonucleotides are shown as below, si-h-PRPS2_001: CTGCAAGATTGCGTCATCA; si-h-PRPS2_002: CCACCAAAG TGTATGCTAT; si-h-PRPS2_003: GAAACACTGCACCAAGATT. The three siRNAs and NC were transfected using Lipofectamine ${ }^{\mathrm{TM}} 3000$ (Invitrogen, Carlsbad, CA, USA) as described in the manufacturer's instructions. After $48 \mathrm{~h}$ of transfection, total RNA or protein was extracted for qRT-PCR or immunoblot analysis as described above. Stable knockdown of PRPS2 in PC-3 cells were achieved by lentivirus vector (GeneChem BioMedical Biotechnology, Shanghai, China). Briefly, cells at 30\% to $40 \%$ confluence were infected by PRPS2 shRNA (CCACCAAAGTGTATGCTAT) lentivirus and empty lentivirus vector, respectively. Thereafter, stable cell lines were selected by puromycin $(5 \mu \mathrm{g} / \mathrm{mL})$ for one week. Then Western blot was conducted to confirm the efficiency of depletion of PRPS2 with short hairpin RNAs.

\section{Cell proliferation assay}

Cell growth was evaluated using the CCK-8 assay (CK04, Dojindo, Japan). PC-3 and DU145 cells were plated in 96-well plates at a density of $2 \times 10^{3}$ per well at $24 \mathrm{~h}$ after transfection. Then CCK-8 reagent (1:100) was added into wells at different time points and incubated for $2 \mathrm{~h}$. The absorbance values at 450 $\mathrm{nm}$ were determined using a microplate reader 
(Dynex Technologies, Inc., Chantilly, VA).

\section{Colony formation Assay}

Cells were seeded onto 6-well plates in triplicate at a density of $1 \times 10^{3}$ cells/well in flat-bottomed six-well plates, and then cultured in complete medium for two weeks. The colonies were fixed in $4 \%$ paraformaldehyde before stained with Wright Giemsa stain (Baso Diagnostics Inc. Zhu Hai, China) for $10 \mathrm{~min}$ for colony counting.

\section{5-ethynyl-20-deoxyuridine assay (EdU) incorporation Assay}

Each group of PCa cells were seeded onto 24 -well plates with a density of $10^{5} /$ well. Then the cells were incubated in the respective media containing $50 \mu \mathrm{M}$ EdU (RiboBio, Guangzhou, China) for $2 \mathrm{~h}$. Cell proliferation was conducted utilizing a Cell-Light $^{\mathrm{TM}}$ EdU DNA Cell Proliferation Kit (RiboBio, Guangzhou, China) according to the manufacturer's protocols. Nuclear DNA was stained with Hoechst 33342 stain $(200 \mu \mathrm{L} /$ well $)$ for $30 \mathrm{~min}$. Images were visualized with an inverted fluorescence microscope at $100 \times$ (Olympus, Tokyo, Japan). The percentage of EdU positive cells (with red fluorescence) to Hoechst nuclear staining cells (with blue fluorescence) was analyzed using ImageJ software to determine the cell proliferation activity.

\section{In vitro cell apoptosis analysis}

Flow cytometry analysis for apoptosis was performed by utilizing Annexin V-FITC Apoptosis Detection Kit (\#KGA108-1, KeyGEN BioTECH) after $48 \mathrm{~h}$ transfection according to the kit's protocol. Cell apoptosis were validated by FACS Caliber FCM (Becton Dickinson Biosciences, San Jose, CA) and histograms generated by using the Flow Jo software package (Tree Star, Inc.). All experiments were analyzed in triplicates.

\section{Cell cycle analysis}

Cells were harvested by tyrisin and washed slightly three times in PBS, then resuspended and cool-fixed at $4{ }^{\circ} \mathrm{C}$ in $70 \%$ ethanol solution overnight. After centrifuge to discard the ethanol we washed cells in PBS three times, then cells were immersed in $10 \mu \mathrm{g} / \mathrm{mL}$ RNase A (Sigma. USA) solution for $30 \mathrm{~min}$ at room temperature. Subsequently, we stained the cells with propidium iodide (PI, $50 \mu \mathrm{g} / \mathrm{mL}$ ) in the dark place. Finally, DNA content of each sample was measured by flow cytometry.

\section{Tumor xenografts in Nude mouse}

For the tumor xenografts experiments, total of 5 $\times 10^{6}$ stable transfected with PRPS2 shRNA (PC-3/sh-PRPS2) or negative control (PC-3/sh-Ctrl)
PC-3 cells in $100 \mu \mathrm{L}$ of PBS were injected subcutaneously into the right flanks of nude male mice ( $\mathrm{n}=8$, per group). Xenograft size was measured using caliper every 3 days, and volumes were evaluated using the formula: $\mathrm{A} \times \mathrm{B}^{2} \times 0.5$ (A represent length and $B$ represent width). At 26 days post injection, mice were euthanized, the tumor specimens were carefully excised, photographed and tissues were preserved for further histologic evaluation (paraffin section) including hematoxylin and eosin (H\&E), IHC and terminal deoxynucleotidyl transferase dUTP nick end labeling (TUNEL). All BALB/c nude mouse experiments were approved by the Institutional Animal Care and Use Committee of Southern Medical University.

\section{TUNEL assays}

All xenograft tumor tissues were fixed with formaldehyde solution for $24 \mathrm{~h}$, dehydrated and embedded in paraffin. Tunnel kit (\#KGA7051, Nanjing KeyGen Biotech Co., Ltd.) was applied to identify apoptosis cells of tumors according to the manufacture's instruction. Briefly, three micrometer-thick deparaffinized sections were deparaffinized, rehydrated and quenched with $1 \%$ proteinase $\mathrm{K}(20 \mathrm{mg} / \mathrm{mL})$ at $37{ }^{\circ} \mathrm{C}$ for $30 \mathrm{~min}$. Subsequently, specimens were washed with PBS and inculcated with TUNEL mixture $(45 \mu \mathrm{L}$ Equilibration Buffer, $1.0 \mu \mathrm{L}$ biotin-11-dUTP, $4.0 \mu \mathrm{L}$ TdT Enzyme, and $50 \mu \mathrm{L}$ reaction buffer) in the dark for $1 \mathrm{~h}$ at $37^{\circ} \mathrm{C}$. After washing with PBS, the specimens were stained with DAPI. Apoptotic cells were stained as red and photographed using fluorescence microscopy at $570 \mathrm{~nm}$.

\section{Statistical analysis}

Statistical analysis was performed with SPSS 20.0 software (SPSS, Chicago, USA). The data are presented as means \pm standard deviation $(\mathrm{SD})$ of at least three repeats. The significance of mean values were analyzed using for continuous variables ANOVA or Student's t-test. Pearson's chi-square test was applied to analyze the clinical variables. $P$ value $<$ 0.05 was considered as statistically significant. ${ }^{*} P<$ $0.05,{ }^{* *} P<0.01$, and $\left.{ }^{* * *} P<0.001\right)$.

\section{Results}

\section{PRPS2 expression levels in PCa tissues and cells}

To detect PRPS2 expression in PCa, we performed IHC analysis of a human prostate tissues microarray. Based on PRPS2 staining levels (Fig. 1A-B), all prostate tissues were divided into two groups: a low expression group $(-$ and +$)$ and a high expression group $(++/+++)$. Results demonstrated 
that PRPS2 was frequently up-regulated in PCa tissue samples $(60 / 78,76.9 \%)$. Whereas the PRPS2 positive rate in the nonneoplastic (normal or adjacent) tissues was significantly lower $(7 / 16,43.8 \%)$. Chi-Square analysis showed that PRPS2 expression levels were higher in PCa tissues than in nonneoplastic (normal or adjacent) tissues (Table $1, \chi^{2}=5.608, P=0.018$ ). Then, we further confirmed the expression of PRPS2 mRNA and protein in five PCa cell lines (PC-3, 22Rv1, DU145, LNCaP, and C4-2) and a noncancerous prostatic epithelial cell (RWPE-1). Two out of five PCa cells had increased PRPS2 protein and mRNA expression level compared with RWPE-1 cells (Fig. 1C). We then assessed the relationship between PRPS2 and clinicopathologic variables. Chi-Square analysis demonstrated PRPS2 protein expression level was positively correlated with clinical stage (Table $1, \chi^{2}=$ 5.114, $P=0.024$ ), and $\mathrm{pT}$ status (Table $1, \chi^{2}=5.966, P=$ 0.015), but was not linked with other clinicopathological features (Table 1). In a word, these findings strongly suggested that PRPS2 expression was associated with PCa invasion and migration. Taken together, these results indicate that PRPS2 was up-regulated in PCa and may be involved in the tumorigenesis of $\mathrm{PCa}$.

\section{Silencing of PRPS2 represses PCa cell proliferation in vitro}

To determine the biological function of PRPS2 in PCa, siRNA targeting PRPS2 (si-PRPS2) was transfected into PC-3 and DU145 cells to inhibit endogenous PRPS2 expression. qRT-PCR and
Western blot confirmed that PRPS2 was markedly decreased in PC-3 and DU145 cells transfected with si-PRPS2 compared with negative control (NC) cells (Fig. 2A). Then we performed CCK-8 and colony formation assays to detect the effect of PRPS2 on cell proliferation. Results demonstrated that PRPS2 knockdown markedly suppressed cell growth and capacity to form colonies of PC-3 and DU145 cells compared with NC cancer cells (Fig. 2B-C, $P<0.001$ ).

Table 1. The correlation between PRPS2 expression and clinicopathological characteristics was analyzed in prostate cancer by $\mathrm{IHC}(\mathrm{n}=78)$.

\begin{tabular}{|c|c|c|c|c|c|}
\hline \multirow[t]{2}{*}{ Variables } & \multirow[t]{2}{*}{ Total N } & \multicolumn{2}{|c|}{ PRPS2 } & \multirow[t]{2}{*}{$\chi^{2}$} & \multirow[t]{2}{*}{$p$ value $^{b}$} \\
\hline & & $\begin{array}{l}\text { High expression } \\
(++/+++, \mathrm{n}, \%)\end{array}$ & $\begin{array}{l}\text { Low expression } \\
(-/+, n, \%)\end{array}$ & & \\
\hline \multicolumn{6}{|l|}{ Type } \\
\hline Normal/BPH & 16 & $7(43.8)$ & $9(56.3)$ & 5.608 & 0.018 \\
\hline PCa & 78 & $60(76.9)$ & $18(23.1)$ & & \\
\hline \multicolumn{6}{|l|}{ Age(years) } \\
\hline$\leq 66^{a}$ & 39 & $26(66.77)$ & $13(33.3)$ & 0.692 & 0.406 \\
\hline$>66$ & 55 & 41 (74.5) & $14(25.5)$ & & \\
\hline \multicolumn{6}{|l|}{ Clinical stage } \\
\hline I- II & 42 & $28(66.7)$ & $14(33.3)$ & 5.114 & 0.024 \\
\hline III- IV & 35 & 31 (88.6) & $4(11.4)$ & & \\
\hline \multicolumn{6}{|l|}{ Primary tumor } \\
\hline $\mathrm{T} 1-\mathrm{T} 2$ & 41 & $27(65.0)$ & $14(34.1)$ & 5.966 & 0.015 \\
\hline T3-T4 & 37 & $33(89.2)$ & $4(10.8)$ & & \\
\hline \multicolumn{6}{|l|}{ Gleason Score } \\
\hline$\leq 7$ & 31 & $23(74.2)$ & $8(25.8)$ & 0.265 & 0.607 \\
\hline$\geq 8$ & 48 & 38 (79.2) & $10(20.8)$ & & \\
\hline
\end{tabular}

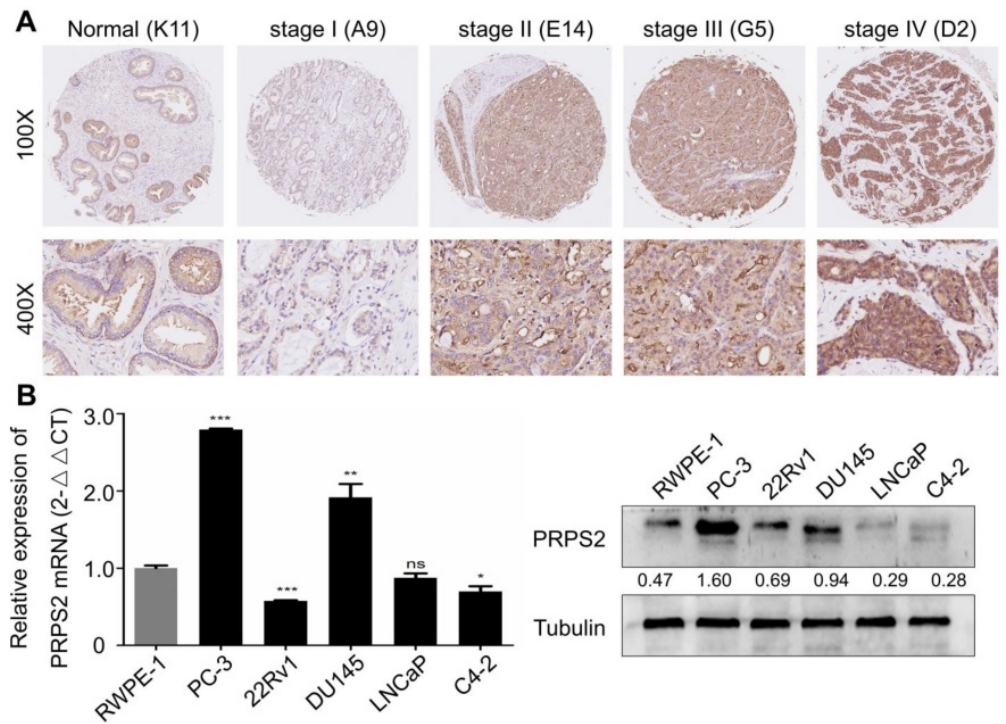

Figure 1. PRPS2 expression in prostate cancer tissues and prostate cancer cell lines. (A) Representative immunohistochemistry images of PRPS2 protein expression from the whole TMA with low intensity (b1) in normal prostate tissue (K11, scale bar $=0.2 \mathrm{~mm})$, with low intensity (b4) in prostate cancer tissue (A9, scale bar $=0.2 \mathrm{~mm}$ ), with intermediate intensity (b3) in prostate cancer tissue (G5, scale bar $=0.2 \mathrm{~mm}$ ), and with high intensity (b2) in prostate cancer tissue (D2, scale bar $=0.2 \mathrm{~mm}$ ). The percentage of PRPS2 positive cells in D2 is $90 \%$, in G5 is $70 \%$, and in A9 is $15 \%$. Therefore, the percentage score of the case in D7 is 3 and its total protein expression score is $3 \times 3=9$. The percentage score of the case in $\mathrm{H} 7$ is 2 and its total protein expression score is $2 \times 2=4$. The percentage score of the case in E4 is 1 and its total protein expression score is $1 \times 1=1$. (B) Western blotting and real-time qPCR analysis of PRPS2 protein and mRNA expression in prostate cancer cell lines and normal prostate epithelial cell lines (RWPE-1). $\left({ }^{*} P<0.05\right.$; ${ }^{*} P<0.01$; ${ }^{* *} P<0.001$ ). PRPS2: Phosphoribosyl pyrophosphate synthetase 2; TMA: tissue microarray. 

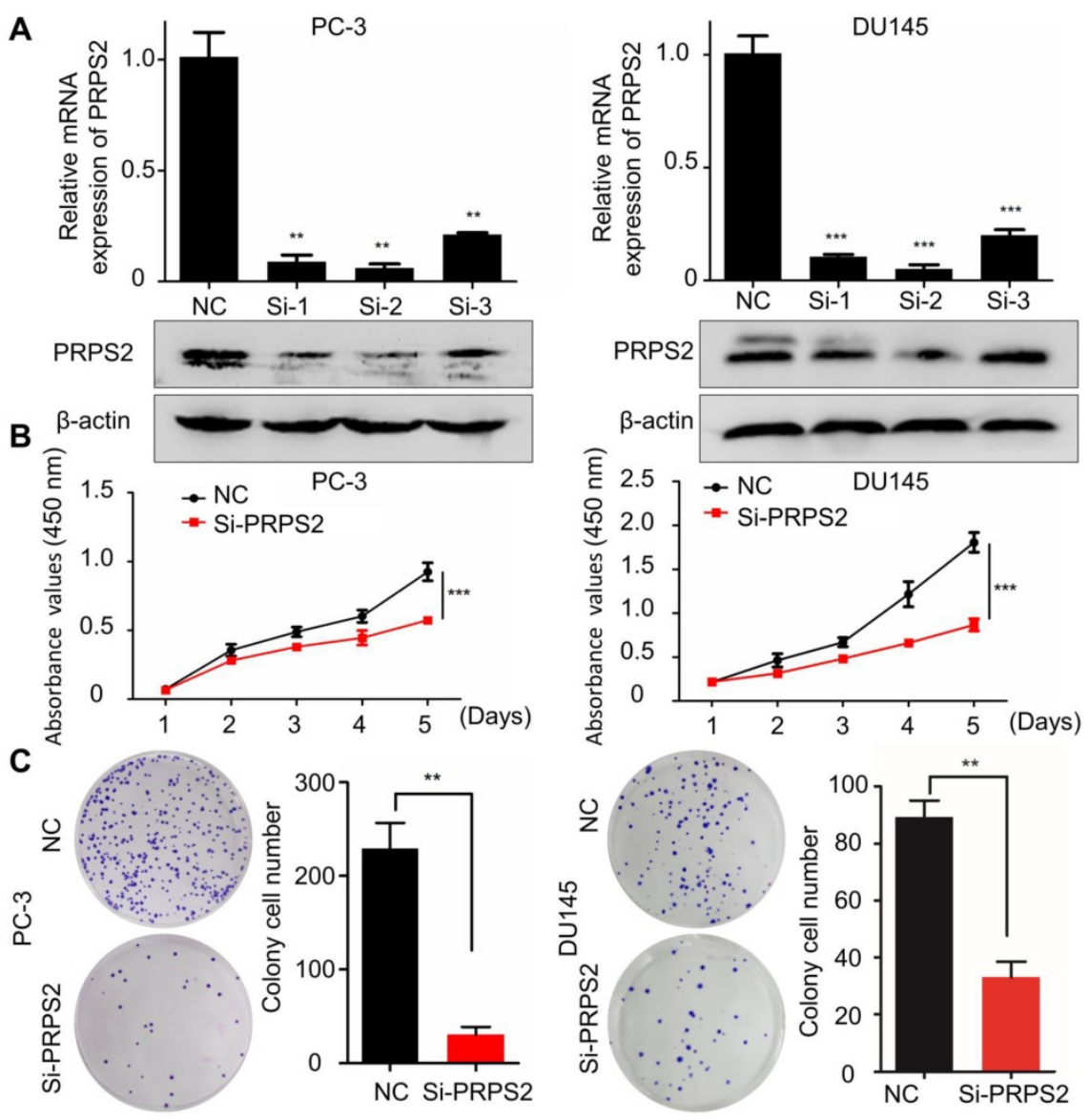

Figure 2. Knockdown of PRPS2 decreased prostate cancer cell growth. (A) The effects of PRPS2 knockdown confirmed by qRT-PCR (top) and Western blotting (bottom). (B) In the CCK-8 assay, cell viability was decreased in si-PRPS2 compared with NC. (C) In the plate colony formation assay, colony formation was obviously decreased in si-PRPS2 cells transfected. ${ }^{* * * *} P<0.001$. NC: negative controls.

\section{PRPS2 knockdown induced cell cycle arrest and apoptosis in PCa cells}

Then we performed flow cytometry to validate whether the PRPS2-depletion suppresses progression through cell cycle. The inhibition of PRPS2 caused a significant increasing of cellular DNA content in $\mathrm{G}_{1}$ phase (Fig. 3A, $\left.P_{P C-3}=0.0012, P_{D U 145}<0.001\right)$. Similarly, EdU incorporation assays revealed that knockdown of PRPS2 markedly inhibited cell proliferation as compared to controls (Fig. 3B, $P_{P C-3}=0.0039, P_{D U 145}=$ 0.0012 ). These results of in vitro experiments indicated that knockdown PRPS2 inhibited the proliferation of PCa cells.

Next, we used flow cytometry to identify the influence of PRPS2 expression on apoptosis. Results showed that apoptosis rates were markedly increased among cells transfected with si-PRPS2 compared with NC cells. Student's $t$-test analysis revealed that the mean total number of apoptosis cells increased from $3.10 \pm 1.15$ to $11.87 \pm 3.70$ and from $2.63 \pm 0.84$ to $9.47 \pm$ 1.55 in response to PRPS2 knockdown in PC-3 cells $(P=0.0173)$ and DU145 cells $(P=0.0026)$, respectively (Fig. $3 C$ ).
To further reveal the potential mechanism underlying this cell cycle arrest and apoptosis promotion by PRPS2 knockdown, we detected the expression of apoptosis-related markers (Bcl-2, Bax, P53, PARP1, caspase-3, caspase-9, cleaved PARP1, cleaved caspase-3 and cleaved caspase-9) and cell cycle related proteins (cyclin D1, P27, and CDK4) by Western blotting. As showed in Figure 3D, the apoptosis-promoting proteins Bax, cleaved PARP1, cleaved caspase-3 and cleaved caspase-9 were substantially increased in response to PRPS2 knockdown compared with NC cancer cells. Conversely, the apoptosis-inhibiting proteins expression of Bcl-2 decreased in si-PRPS2-transfected PCa cells. With regards to cell cycle related proteins silencing of PRPS2 significantly increased cyclin-dependent kinase inhibitor P27, whereas cyclin D1 which functions as a mitogenic sensor and allosteric activator of CDK4/ 6 decreased remarkably. Therefore, these results indicated that PRPS2 depletion activated intrinsic apoptosis by activating $\mathrm{PARP} / \mathrm{Bcl} 2 /$ Caspase pathways and induced cell cycle $\mathrm{G}_{1}$ arrest at least partly attributed to cyclin-dependent kinase. 
A
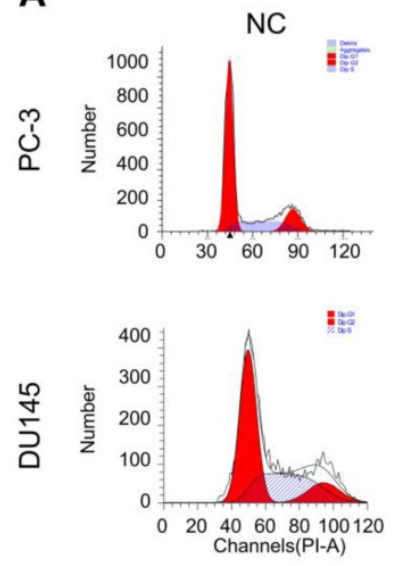

B $\frac{\mathrm{PC}-3}{\mathrm{NC} \quad \mathrm{Si-PRPS2}}$
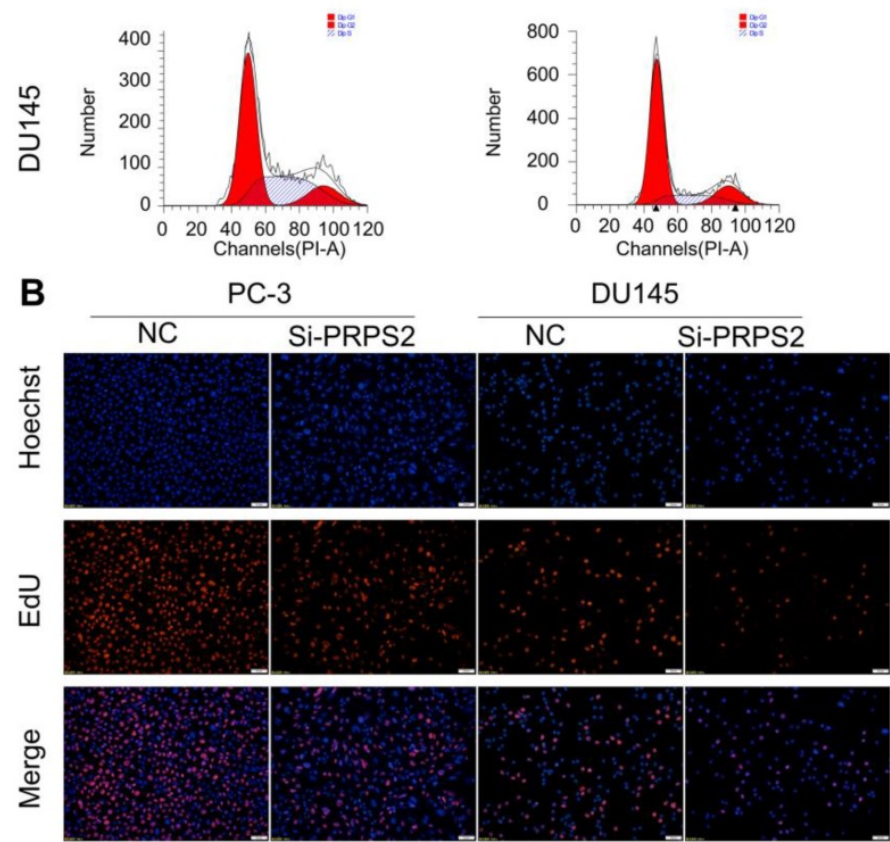

DU145
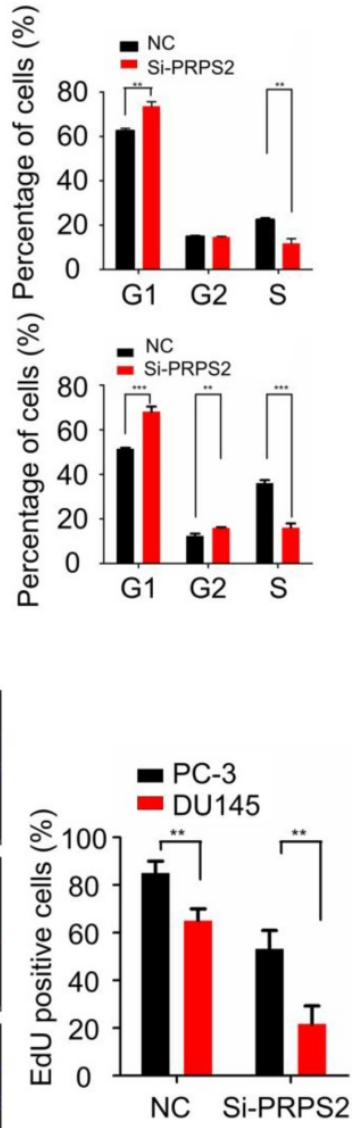

C
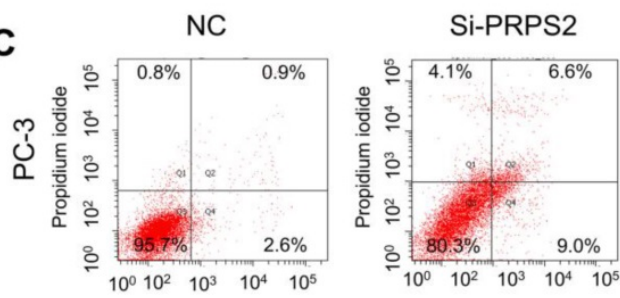

D

sh-PRPS2
NC

$\frac{\text { PC-3 }}{-+} \quad \frac{\text { DU145 }}{-}+$

PRPS2

CyclinD1

P27

CDK4
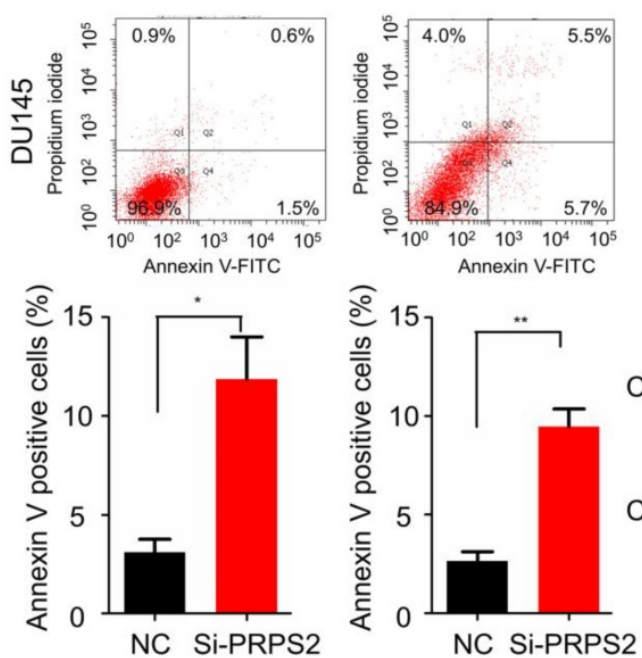

Cleaved PARP

PARP

p53

Bax

Bcl-2

Cleaved Caspase-9

Caspase-9

Cleaved Caspase-3

Caspase-3

$\beta$-actin
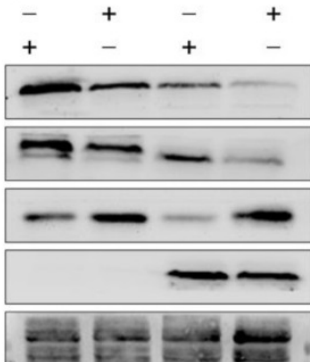

$-m=2$
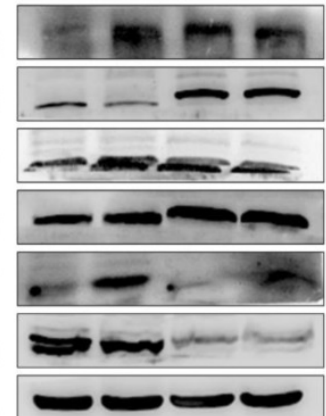

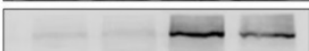

Figure 3. Knockdown of PRPS2 induced prostate cancer cell apoptosis and cell cycle arrest. (A) PRPS2 knockdown induced PCa cell cycle arrested in $G_{0} / G_{1}$ phase. (B) Representative micrographs (left) and quantification (right) of EdU incorporation assay. ${ }^{* *} P<0.01,{ }^{* * * *} P<0.001$. (C) PRPS2 knockdown increased apoptosis rate. ${ }^{*} P<0.05$. (D) Western blot showed the protein levels of cell apoptosis-related proteins (cleavage of caspase-3, caspase-9, PARP, Bcl-2, Bax and p53) and cell cycle-related proteins (P27, CDK4 and cyclin DI) after down-regulated expression of PRPS2 in PC-3 (left) and DU145(right) cells. $\beta$-actin was served as loading control. NC, negative control. 
A

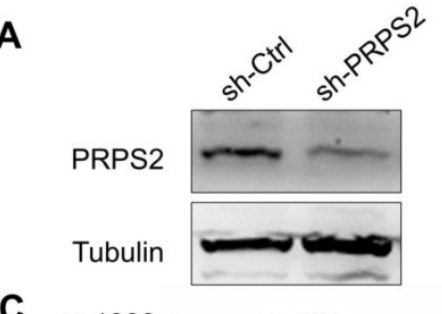

C

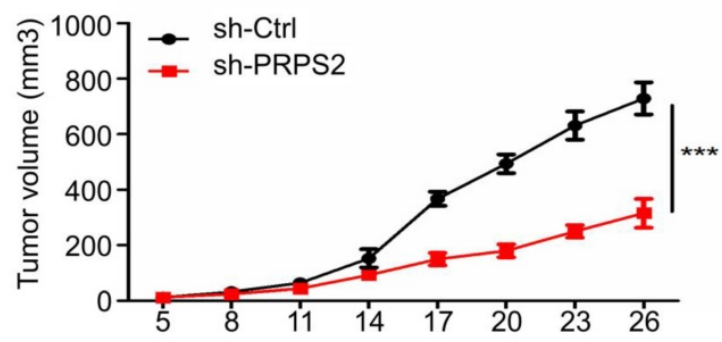

E

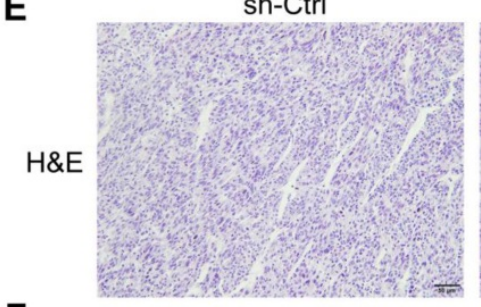

$\mathbf{F}$
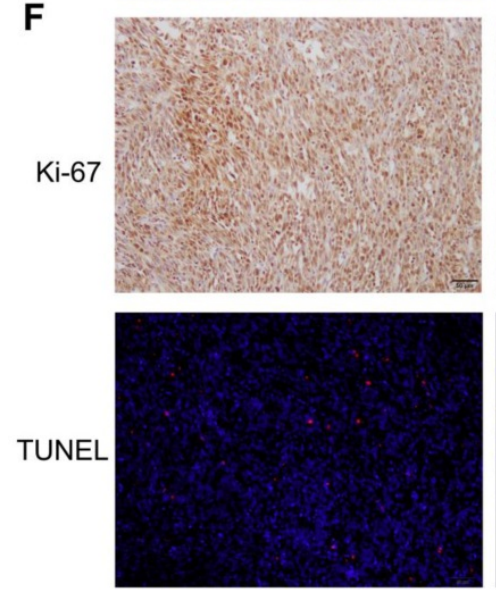

sh-PRPS2
B

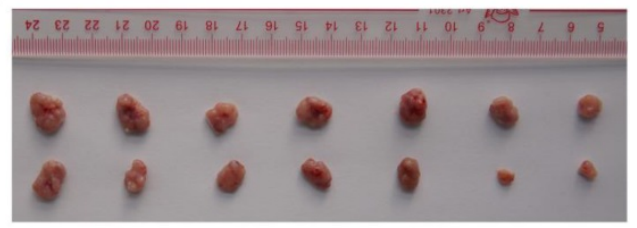

D

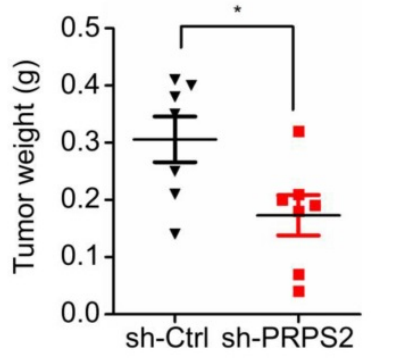

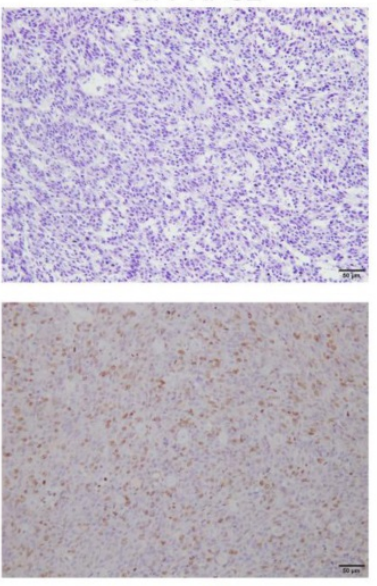
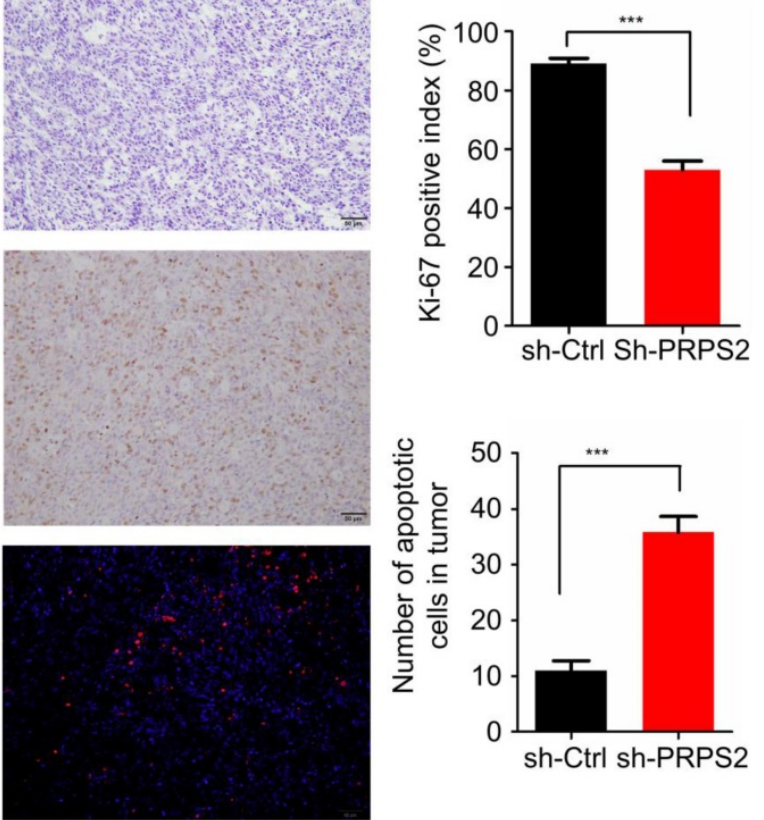

Figure 4. Knockdown of PRPS2 inhibits xenograft tumor growth in vivo. (A) PRPS2 shRNA effectively blocked its protein expression in PC-3 cells. Cells were stably transfected with scramble (sh-Ctrl) or PRPS2 shRNA (sh-PRPS2). PRPS2 protein levels were normalized to $\alpha$-Tubulin. (B) Gross observation of xenograft tumor size in NOD/SCID mice. (C and D) Silencing of PRPS2 inhibited the tumor growth, including tumor volume $(P<0.001)$ and weight $(P=0.028, \mathrm{n}=6)$. $(E)$ H\&E-stained paraffin-embedded sections obtained from xenografts of PC-3 cells. (F) Top: Immunohistochemical analysis of Ki-67 in the xenografts; Bottom: The apoptosis in tumor tissues was evaluated by TUNEL assay $(\times 200)$. Graphical illustrated the quantification of Ki-67 and TUNEL positive cells percentage. ${ }^{*} * \mathrm{P} P<0.001$.

\section{Knockdown of PRPS2 inhibited xenograft tumor growth in vivo}

To evaluate the impact of PRPS2 knockdown on tumor growth in vivo, we established a subcutaneous xenograft tumor model in athymic nude mice by injecting PC-3 cells infected with shRNA targeting PRPS2 or NC PC-3 cells. Results from Western blotting showed an obvious reduction of PRPS2 expression in PC-3 cells transfected with sh-PRPS2 compared with NC cells (Fig. 4A). As expected, cells with PRPS2 knocked down formed slower-growing xenografts compared with NC cells (Fig. 4B-C, $P<0.001)$. Correspondingly, tumor weight in PRPS2 depletion group was lighter than the control group (Fig. $4 \mathrm{D}, P=0.028$ ). H\&E staining revealed histopathological features of the xenograft tumor tissues (Fig. 4E). Moreover, IHC results of the xenograft tumor tissues demonstrated that the expression of Ki-67 proliferation antigen in xenografts of sh-PRPS2 cells was significantly decreased (Fig. 4F, upper panel, $P<0.001)$. TUNEL staining also showed an apoptosis enhancement of tumor cells after PRPS2 knockdown (Fig. 4F, lower panel, $P<0.001$ ). All these results further indicated that PRPS2 knockdown suppressed cell growth at least partially mediated by the elevated intrinsic apoptosis of PCa cells. 
A
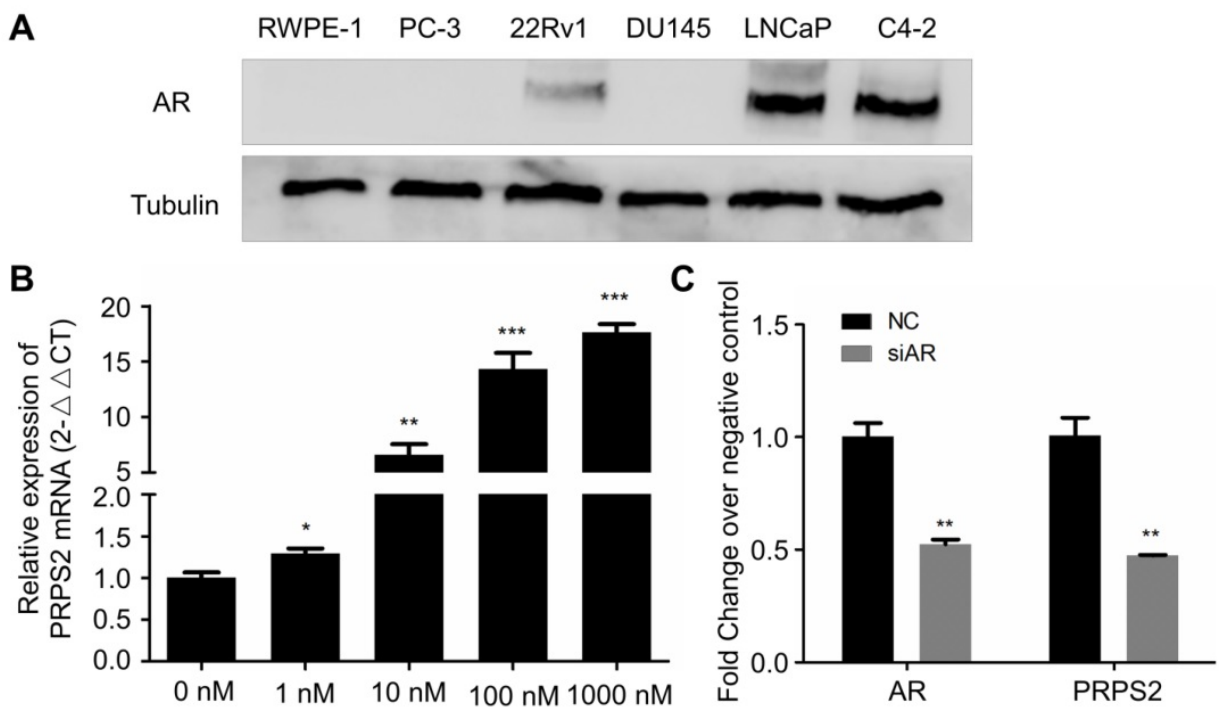

Figure 5. PRPS2 was an androgen-responsive gene. (A) Western blotting of AR protein expression in prostate cancer cell lines and normal prostate epithelial cell lines (RWPE-1). (B) qRT-PCR analysis of PRPS2 expression with androgen-starved for $72 \mathrm{~h}$ and treated with DHT $48 \mathrm{~h}$ in dose series of $0 \mathrm{nM}, 1 \mathrm{nM}, 10 \mathrm{nM}, 100$ $\mathrm{nM}, 1000 \mathrm{nM}$ in LNCaP. The expression of PRPS2 after DHT stimulation was normalized to the expression without DHT stimulation. (C) The efficiency of siAR on the expression of AR was confirmed by $\mathrm{qRT}-\mathrm{PCR}$ and the expression of PRPS2 was tested after transfection of siAR compared with NC in LNCaP cells. Data are presented as the mean \pm SD $(n=3) .\left({ }^{*} P<0.05\right.$; **P $<0.01$; **** $\left.P<0.001\right)$.

\section{Androgen treatment could induce PRPS2 expression}

Emerging evidence had proved that androgen receptor (AR) played the most vital roles in PCa development through regulating androgen-responsive genes [12]. Interestingly, our previous study revealed that PRPS2 expression involved in sertoli-cell only syndrome (SCOS) and inhibits the apoptosis of sertoli cells via the p53/Bcl-2/caspases signaling pathway [9]. Besides, testis AR is exclusively upregulated in SCOS than in normal testis [13]. Thus, we hypothesized PRPS2 may be also an androgen regulated gene. To confirm this, we employed dose-dependent DHT treatment to detect whether the expression of PRPS2 is androgen-related in LNCaP cells. Firstly, we confirmed the expression of $\mathrm{AR}$ was decisive in 22Rv1, LNCaP and C4-2, whereas PC-3, DU145 and RWPE-1 cells demonstrated negative expression of AR (Fig. 5A). Then qRT-PCR analysis of PRPS2 expression with androgen-starved for $72 \mathrm{~h}$ and treated with DHT for $48 \mathrm{~h}$ in LNCaP cells. Results demonstrated that PRPS2 expression increased 17.6fold (Fig. 5B, $P<0.001$ ) after high dose of DHT $(1000 \mathrm{nM})$ stimulation compared with control. Moreover, we also detected that PRPS2 expression was reduced by $53 \%$ over negative control (NC) following si-AR transfection (Fig. $5 C, P=0.0026$ ). The results implied that PRPS2 may be an androgen-responsive gene.

\section{Discussion}

In this study, we found that PRPS2 was upregulated in PCa tissues compared with normal prostate tissues. Moreover, patients with higher clinical stage and pT status exhibited increased PRPS2 expression. Then we performed loss of function experiments in vitro and revealed that silencing of PRPS2 could remarkably suppress the cell proliferation, which might attribute to cell cycle arrest and PRAP-caspase dependent cancer cell apoptosis. In addition, knockdown of PRPS2 drastically inhibited the xenograft tumor growth in vivo. These results suggested that PRPS2 may involve in progression and aggressiveness in PCa.

The ability to modify metabolic output to fulfill the biosynthetic and bioenergetic demands of cell growth and proliferation is a defining feature of cancer cells [14, 15]. The Myc oncogene regulated several cellular responses including promoting protein synthesis and nucleotide synthesis, imperative for sustaining cancer cell proliferation. PRPS2 is a key rate-limiting enzyme within the urine synthesis pathway which has been reported to be translational upregulated by Myc-driven hyperactivation of protein synthesis [7]. There are three isoforms of homo-sapiens PRPS family on record, the amino acid homology of PRPS1 and PRPS2 is approximately 95\% [5], PRPS can regulate cancer metabolism and promotes nucleotide synthesis under tumor energy stress [16]. As an important rate-limiting enzyme of the pentose-phosphate pathway, PRPS2 boosts increased nucleotide biosynthesis via specialized cis-regulatory elements with the PRSP 5'UTR [7]. In addition, PRPS2 was confirmed to catalyze the reaction of converse ribose 
5-phosphate into 1-5-phosphoribosyl pyrophosphate (PRPP) which is irreversible $[17,18]$. Recently studies demonstrated that PRPS2 was involved in cancer cell proliferation and metastasis of neuroblastoma malignancy, which was regulated by both MYCN and activating enhancer binding protein-4 (TFAP4) [19]. Moreover, pervious study has suggested that PRPS2 may act as an oncogene in PCa on account of PRPP is a key precursor of purine synthesis [20]. Meanwhile, lowering serum uric acid levels in PCa was regarded as therapeutically beneficial [21]. However, the biological function of PRPS2 in PCa remains largely unknown. Thus, we speculated the occurrence and progress of $\mathrm{PCa}$ may be closely related to the unusually exuberant nucleic acid metabolism that was regulated by PRPS2, which provided new evidence for us to learn the abnormal metabolism of PCa cells. As expected, we identified that PRPS2 may be act as an oncogene role to facilitate the growth of PCa cells.

It has become a truism that sustaining proliferation is an important hallmark of cancer [22]. c-MYC has been founded an elevated expression in PCa and multiple studies shown c-MYC can induce tumor cells proliferation and regulate cell cycle though MYC-responsive genes [7, 23-25]. Cell cycle genes expression level has been founded that associate with the timing of PCa metastasis, as an independent factor [26]. Thus, cell cycle related genes or proteins have significant prognostic value in patients with PCa. Furthermore, it has been reported that glucose deprivation can drive brain tumorigenesis via c-MYC/AMPK direct phosphorylation of PRPS1 S180 and PRPS2 S183 [16], indicated PRPS2 is a c-MYC pathway downstream gene. Myc-overexpressing cells lacking PRPS2 have a decreased ability to increase protein synthesis, possibly because of the reduced nucleotide production required to synthesize ribosomes. Thus, in this study, our data discovered that PRPS2 knockdown significantly upregulated cyclin-dependent kinase inhibitor p27 but downregulated $\mathrm{G}_{1} / \mathrm{S}$-specific protein cyclin D1 both in PC3 and DU145 cells, indicated that PRPS2 may be a potential druggable target for c-MYC responsive cell cycle checkpoint pathway in PCa.

Resisting apoptosis is common in all kinds of tumors. Dysregulation of apoptosis is also regarded as a hallmark of PCa [27-29]. Hence, restore the normal apoptotic cell death in PCa is a critical treatment strategy [30-32], such as androgen deprivation therapy [33]. However, after current ADT therapy mostly advanced PCa development to castration-resistant prostate cancer (CRPC), force physicians to find different strategies according to novel AR-related mechanisms [34]. Purinergic receptor has been reported to involve in mitochondrial apoptosis via either androgen dependent or independent PCa cells [35]. Our study demonstrated that PRPS2 enhanced the ability of apoptosis resistant both in PC-3 and DU145. Further, we founded that the protein expression levels of Bax, cleaved PARP and cleaved caspase-3 in PCa cells were significantly increased in PRPS2 knockdown than controlled groups. Indicated that PRPS2 boosts PCa development via inhibited Bax-caspase9/3-PARP dependent apoptosis, which has not been reported yet.

Interestingly, our previous study has revealed that PRPS2 inhibits the apoptosis of TM4 Sertoli cells via the caspases depended signal pathway [9]. Besides, testis AR is exclusively overexpression in Sertoli cells only syndrome than in normal testes [13]. Recent literatures reported a gene aberrations analysis in PCa before and after proceeding ADT treatment suggested MYC may involvement in CRPC development [36]. These studies imply a relationship between AR and MYC related genes. Here, our results demonstrated that DHT stimulation enhanced PRPS2 expression in a dose-dependent manner, which further indicated that PRPS2 may be a testosterone-sensitive enzyme and partly contributed to the occurrence and progress of PCa. However, whether there is a regulatory relationship between abnormal PRPS2 and androgen receptors still needs further studies.

\section{Conclusion}

In summary our study has presented that high PRPS2 expression correlated with the aggressive clinical feature of PCa. Moreover, PRPS2 knockdown efficiently inhibited PCa cell proliferation partly owes to induce cell cycle arrest and facilitate caspase-dependent apoptosis. These results indicated that PRPS2 may play a crucial role in the progression and aggressiveness of PCa, interfering with PRPS2 signaling may develop therapeutic value against PCa with high expression of PRPS2.

\section{Abbreviations}

5'-UTR: 5'- Untranslated Regions; ADT: Androgen deprivation therapy; AR: Androgen receptor; CRPC: Castration-resistant prostate cancer; DAB: Diaminobenzidine; DHT: Double hydrogen testosterone; EdU: 5-ethynyl-20-deoxyuridine assay; EGF: Epidermal growth factor; IHC: Immunohistochemistry; KSFM: Keratinocyte serum free medium; PI: Propidium iodide; PRPP: Phosphoribosyl pyrophosphate; PRPS: Phosphoribosyl pyrophosphate synthetases; PRPS2: Phosphoribosyl pyrophosphate synthetases 2; PRTE: Pyrimidine-rich 
translational element; TMA: Tissue microarray; TNM: Tumor node metastasis.

\section{Supplementary Material}

Supplementary figure 1.

http://www.jcancer.org/v11p1027s1.pdf

Supplementary table 1.

http://www.jcancer.org/v11p1027s2.xlsx

\section{Acknowledgements}

This study was supported by grants from the National Natural Science Foundation of China (No. 8177110496), the Science and Technology planning Project of Guangzhou (No. 201803010014), China Postdoctoral Science Foundation funded project (NO.2018M643126) and Guangdong medical research fund project (NO. A2017388).

\section{Authors' contributions}

MXM and ZYG: participated in study design and coordination, analysis and interpretation of data, material support for obtained funding, and supervised study. QH, LDJ and TX: performed most of the experiments and statistical analysis and drafted the manuscript. Other author: carry out the experiment and sample collection. All authors have read and approved the final version of the manuscript, and agree with the order of presentation of the authors.

\section{Competing Interests}

The authors have declared that no competing interest exists.

\section{References}

1. Bray F, Ferlay J, Soerjomataram I, et al. Global cancer statistics 2018: GLOBOCAN estimates of incidence and mortality worldwide for 36 cancers in 185 countries. CA Cancer J Clin. 2018; 68: 394-424.

2. Guillaumier S, Peters M, Arya M, et al. A Multicentre Study of 5-year Outcomes Following Focal Therapy in Treating Clinically Significant Nonmetastatic Prostate Cancer. Eur Urol. 2018; 74: 422-9.

3. Zhou X, Li Q, He J, et al. HnRNP-L promotes prostate cancer progression by enhancing cell cycling and inhibiting apoptosis. Oncotarget. 2017; 8: 19342-53.

4. Cao S, Ma T, Ungerleider N, et al. Circular RNAs add diversity to androgen receptor isoform repertoire in castration-resistant prostate cancer. Oncogene. 2019; [Epub ahead of print].

5. Becker MA, Heidler SA, Bell GI, et al. Cloning of cDNAs for human phosphoribosylpyrophosphate synthetases 1 and 2 and $X$ chromosome localization of PRPS1 and PRPS2 genes. Genomics. 1990; 8: 555-61.

6. Taira M, Iizasa T, Yamada K, et al. Tissue-differential expression of two distinct genes for phosphoribosyl pyrophosphate synthetase and existence of the testis-specific transcript. Biochim Biophys Acta. 1989; 1007: 203-8.

7. Cunningham JT, Moreno MV, Lodi A, et al. Protein and nucleotide biosynthesis are coupled by a single rate-limiting enzyme, PRPS2, to drive cancer. Cell. 2014; 157: 1088-103.

8. Zhang F, Patel DM, Colavita K, et al. Arginylation regulates purine nucleotide biosynthesis by enhancing the activity of phosphoribosyl pyrophosphate synthase. Nat Commun. 2015; 6: 7517.

9. Lei B, Wan B, Peng J, et al. PRPS2 Expression Correlates with Sertoli-Cell Only Syndrome and Inhibits the Apoptosis of TM4 Sertoli Cells. J Urol. 2015; 194: 1491-7.
10. Lv D, Wu H, Xing $\mathrm{R}$, et al. HnRNP-L mediates bladder cancer progression by inhibiting apoptotic signaling and enhancing MAPK signaling pathways. Oncotarget. 2017; 8: 13586-99.

11. Hu ZY, Liu YP, Xie LY, et al. AKAP-9 promotes colorectal cancer development by regulating Cdc42 interacting protein 4. Biochim Biophys Acta. 2016; 1862: 1172-81.

12. Yin $\mathrm{Y}, \mathrm{Li} \mathrm{R}, \mathrm{Xu} \mathrm{K}$, et al. Androgen Receptor Variants Mediate DNA Repair after Prostate Cancer Irradiation. Cancer Res. 2017; 77: 4745-54.

13. Loukil LH, Boudawara TS, Ayadi I, et al. High androgen receptor immunoexpression in human "Sertoli cell only" testis. Arch Inst Pasteur Tunis. 2005; 82: 47-51.

14. Cairns RA, Harris IS, Mak TW. Regulation of cancer cell metabolism. Nat Rev Cancer. 2011; 11: 85-95.

15. Vander Heiden MG, Lunt SY, Dayton TL, et al. Metabolic pathway alterations that support cell proliferation. Cold Spring Harb Symp Quant Biol. 2011; 76: 325-34.

16. Qian X, Li X, Tan L, et al. Conversion of PRPS Hexamer to Monomer by AMPK-Mediated Phosphorylation Inhibits Nucleotide Synthesis in Response to Energy Stress. Cancer Discov. 2018; 8: 94-107.

17. Visentin LP, Hasnain S, Gallin W. Ribosomal protein S1/S1A in bacteria. FEBS Lett. 1977; 79: 258-63.

18. Li S, Lu Y, Peng B, et al. Crystal structure of human phosphoribosyl pyrophosphate synthetase 1 reveals a novel allosteric site. Biochem J. 2007; 401: 39-47.

19. Xue C, Yu DM, Gherardi S, et al. MYCN promotes neuroblastoma malignancy by establishing a regulatory circuit with transcription factor AP4. Oncotarget. 2016; 7: 54937-51.

20. Hove-Jensen B. Mutation in the phosphoribosyl pyrophosphate synthetase gene (prs) that results in simultaneous requirements for purine and pyrimidine nucleosides, nicotinamide nucleotide, histidine, and tryptophan in Escherichia coli. J Bacteriol. 1988; 170: 1148-52.

21. Sangkop F, Singh G, Rodrigues E, et al. Uric acid: a modulator of prostate cells and activin sensitivity. Mol Cell Biochem. 2016; 414: 187-99.

22. Hanahan D, Weinberg RA. Hallmarks of cancer: the next generation. Cell. 2011; 144: 646-74.

23. Mannava S, Grachtchouk V, Wheeler LJ, et al. Direct role of nucleotide metabolism in C-MYC-dependent proliferation of melanoma cells. Cell Cycle. 2008; 7: 2392-400.

24. Zhang W, Liu B, Wu W, et al. Targeting the MYCN-PARP-DNA Damage Response Pathway in Neuroendocrine Prostate Cancer. Clin Cancer Res. 2018; 24: 696-707.

25. Elliott B, Millena AC, Matyunina L, et al. Essential role of JunD in cell proliferation is mediated via MYC signaling in prostate cancer cells. Cancer Lett. 2019; 448: 155-67.

26. Rubicz R, Zhao S, April C, et al. Expression of cell cycle-regulated genes and prostate cancer prognosis in a population-based cohort. Prostate. 2015; 75: 1354-62

27. Pistritto G, Trisciuoglio D, Ceci C, et al. Apoptosis as anticancer mechanism: function and dysfunction of its modulators and targeted therapeutic strategies. Aging. 2016; 8: 603-19.

28. Baig S, Seevasant I, Mohamad J, et al. Potential of apoptotic pathway-targeted cancer therapeutic research: Where do we stand? Cell Death Dis. 2016; 7: e2058.

29. Yang C, Zhang W, Wang J, et al. Effect of docetaxel on the regulation of proliferation and apoptosis of human prostate cancer cells. Mol Med Rep. 2019.

30. Kulik G. ADRB2-Targeting Therapies for Prostate Cancer. Cancers. 2019; 11: 358 .

31. Wang J, Nasser MI, Adlat S, et al. Atractylenolide II Induces Apoptosis of Prostate Cancer Cells through Regulation of AR and JAK2/STAT3 Signaling Pathways. Molecules. 2018; 23: 3298.

32. Foo SL, Yap G, Cui J, et al. Annexin-A1 - A Blessing or a Curse in Cancer? Trends Mol Med. 2019; 25: 315-327.

33. Ferrari N, Granata I, Capaia M, et al. Adaptive phenotype drives resistance to androgen deprivation therapy in prostate cancer. Cell Commun Signal. 2017; 15: 51.

34. Pakula H, Xiang D, Li Z. A Tale of Two Signals: AR and WNT in Development and Tumorigenesis of Prostate and Mammary Gland. Cancers. 2017; 9: 14.

35. Aghaei M, Karami-Tehrani F, Panjehpour M, et al. Adenosine induces cell-cycle arrest and apoptosis in androgen-dependent and -independent prostate cancer cell lines, LNcap-FGC-10, DU-145, and PC3. Prostate. 2012; 72: 361-75.

36. Kaltz-Wittmer C, Klenk U, Glaessgen A, et al. FISH analysis of gene aberrations (MYC, CCND1, ERBB2, RB, and AR) in advanced prostatic carcinomas before and after androgen deprivation therapy. Lab Invest. 2000; 80: 1455-64. 